\title{
Past, present and future of information and knowledge sharing in the construction industry: towards semantic service-based e-construction?
}

Yacine Rezgui $^{[1]}$, Stefan Boddy ${ }^{[2]}$, Matthew Wetherill ${ }^{[2]}$, Grahame Cooper ${ }^{[3]}$

[1] Cardiff University, Queen's Buildings, The Parade, Cardiff, CF24 3AA, Wales, UK.

[2] Salford Centre for Research and Innovation in the Built and Human Environment, Maxwell Building, University of Salford, The Crescent, Salford, Greater Manchester, M5 4WT, UK

[3] Informatics Research Institute, Maxwell Building, University of Salford, The Crescent, Salford, Greater Manchester, M5 4WT, UK.

\author{
Corresponding author: \\ Cardiff University \\ Queen's Buildings \\ The Parade \\ CARDIFF CF24 3AA \\ Wales, UK.
}

Email: RezguiY@cardiff.ac.uk 


\begin{abstract}
The paper reviews product data technology initiatives in the construction sector and provides a synthesis of related ICT industry needs. A comparison between (a) the data centric characteristics of Product Data Technology (PDT) and (b) ontology with a focus on semantics, is given, highlighting the pros and cons of each approach. The paper advocates the migration from data-centric application integration to ontology-based business process support, and proposes inter-enterprise collaboration architectures and frameworks based on semantic services, underpinned by ontology-based knowledge structures. The paper discusses the main reasons behind the low industry take up of product data technology, and proposes a preliminary roadmap for the wide industry diffusion of the proposed approach. In this respect, the paper stresses the value of adopting alliance-based modes of operation.
\end{abstract}

\title{
Keywords:
}

Product Data, IFCs, Ontology, CAD, Web Services, e-Process.

\section{Introduction}

Construction is a knowledge intensive industry characterized by its unique work settings and virtual organization like modus operandi [54]. The Construction sector is fragmented and the major consequence is the difficulty to communicate effectively and efficiently among partners during a building project or between clients and suppliers of construction products. Several initiatives led by standardisation and / or industry consortia have developed data / product models aimed at facilitating data and information exchange between software applications. These efforts include STEP [33] and the Industry Foundation Classes (IFCs) [32]. Several other initiatives at a national 
and European level have developed dictionaries, thesauri, and several linguistic resources focused on Construction terms to facilitate communication and improve understanding between the various stakeholders operating on a project or across the product supply chain. However, these initiatives tend to be country specific and not adapted to the multi-national nature of the sector. Also, given the vast scope of Construction, these semantic resources tend to be specialized for dedicated applications or engineering functions, e.g. product libraries and HVAC (Heating, Ventilation and Air Conditioning), respectively.

A comprehensive literature review targeting Computer Integrated Construction (CIC) was reported by the authors in [8]. This review reveals a strong focus on data and application integration research. It is argued that whilst valuable, such research and the software solutions it yields fall short of the potential for CIC. Thus the authors call for re-focussing CIC research on the relatively under-represented area of semantically described and coordinated process oriented systems to better support the kind of short term virtual organisation that typifies the working environment in the construction sector. Moreover, the review provides a Framework that illustrates the CIC research landscape (Figure 1). A two dimensional representation is used, developed with respect to two axes: (a) Semantic Focus - this axis spans the whole spectrum of past, existing, and future applications with underlying semantics ranging from data structures conveyed through data models to rich-semantic representations through ontology; (b)

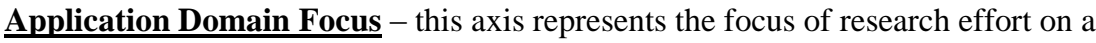
continuum from application and API (Application Programming Interface) centric, to process and people centric. 
Figure 1: Computer Integrated Construction research landscape [8]

This paper focuses on the right-bottom quadrant of Figure 1 and argues the case for a change of emphasis from data and object centric applications to high-level process driven semantic services. It builds on the results of a wide consultation led by the authors in the context of the EU funded ROADCON project [58] that resulted in (a) comprehensive industry requirements, (b) an ICT vision, and (c) the first ICT roadmap for the Construction industry. In fact, the authors' research over the last decade (as illustrated in Figure 2) has evolved from advanced data and information management solutions [57], applied later in the context of CAD [13], to advanced knowledge management systems, articulated around the use of an ontology [40] [53], and deployed in distributed environments [52] [45]. Moreover, the emphasis on knowledge infused applications using service-oriented architectures has been made and reported in [46] [47] [48] [50].

As such, the paper makes four main contributions: (a) it outlines and discusses the various approaches to integration adopted by the Construction IT community; (b) it argues the case for ontologies as a means to address limitations faced by existing Product / Data model standards; (c) it puts ontologies into context by promoting a service-oriented view of the world; and (d) it discusses adoption and diffusion issues informed by existing STEP / IFC initiatives and proposes a way forward.

The paper is organised as follows. First, the methodology that underpins the proposed research is presented in section 2. Section 3 reviews two decades of product data research from early product models to current so called Building Information Models 
(BIM). A critical discussion on product data technology is then provided in section 4, arguing the case for knowledge-rich ontology. Section 5 discusses industry adoption and diffusion of IFCs, and identifies key requirements for the development and adoption of a construction ontology. Section 6 then discusses how an ontology can play a pivotal role in enabling seamless inter-working and interoperability between diverse webenabled applications, namely web-services, and identifies essential requirements for supporting dynamic, long lasting, processes as experienced during the design stage of a building. Section 7 provides a description of the technical infrastructure followed finally, in section 8 , by proposals for a staged roadmap, inspired from the ROADCON project [58], to help the industry migrate from current product data centric applications to semantic ontology-enabled services.

\section{Methodology}

The research presented in the paper adopts a reflective practice approach underpinned by a participatory action research methodology [21]. There are two sorts of reflection: reflection-in-action and reflection-on-action. The former involves a level of awareness throughout the action, while in the latter reflection is done after the action or event. The paper adopts a reflection-on-action approach. In fact, several case study projects that span a period of over ten years form the focus of this study. These are depicted in Figure 2. The value of a person's reflection can be greatly enhanced by a greater understanding of the process. This allows the participants to create their own knowledge and theory relevant to their own specific situation.

Figure 2: Authors' involvement in national and UK CIC initiatives 
The paper will reflect on the authors' 15 years active involvement in national (UK) and EU funded research (as illustrated in Figure 2) and propose a re-focussing of CIC research on high-level process driven semantic services.

\section{From simple Product data to complex building information models}

Although the manual referencing of paper based product data and building design has existed for centuries, it was the increasing use of CAD facilities in design offices from the early 1980s which prompted the first efforts in electronic integration and sharing of building information and data [8]. Here, the ability to share design data and drawings electronically through either proprietary drawing formats or via later de facto standards such as DXF (Drawing / Data Exchange Format), together with the added dimension of drawing layering had substantial impacts on business processes and workflows in the construction industry [17]. Although in these early efforts, sharing and integration was mainly limited to geometrical information [11], effectively the use of CAD files was evolving towards communicating information about a building in ways that a manually draughted or plotted drawing could not [2].

This evolution continued with the introduction of object-oriented CAD in the early 1990s by companies such as AutoDesk, GraphiSoft, Bentley Systems etc. Data “objects" in these systems (doors, walls, windows, roofs, plant and equipment etc.) stored non-graphical data about a building and the third party components which it comprises "product data", in a logical structure together with the graphical representation of the building [15] [2]. These systems often supported geometrical 
modelling of the building in three dimensions, which helped to automate many of the draughting tasks required to produce engineering drawings.

When combined with the increasing ubiquity of electronic networking and the Internet, this allowed many companies to collaborate and share building information and data which in turn lead to new ways of communicating and working [15] [10]. The opportunities presented by the move towards collaborative working and information sharing encouraged a number of research projects in the early 1990's, which aimed to facilitate and provide frameworks to encourage the migration from document centred approaches towards model based, integrated systems: CONDOR [51]; COMMIT [57] being examples. Similarly, the OSMOS [52] research project aimed to develop a technical infrastructure which empowered the construction industry to move towards a computer, integrated approach.

It became clear that in order to take best advantage of the potential for CAD and object / product model integration, there was a need for more coordinated standards which would simplify and encourage its uptake [29]. These standards defining efforts came in the form of the STEP application protocols for construction [32] [24] [39]. This work, inspired by previous work primarily in aerospace and automotive fields, formed part of ISO 10303, the International Standard for the Exchange of Product Model Data.

Latterly, the International Alliance for Interoperability defined the Industry Foundation Classes, a set of model constructs for the description of building elements. Preceding and in some cases concurrent with this work, the research community produced several Comment [i1]: Academic deleted as per Reviewer 4, comment 4 integrated model definitions including GARM [27], the AEC Building Systems Model [63], ATLAS [9], the RATAS model [5], OPIS [25], and the COMBINE Integrated 
Data Model [32]. These research efforts tended to propose a data model and also provide a suite of tools to manipulate the model (as a proof of concept), or a central database to serve model elements to other applications used in the construction project process via some form of adapter [6] [7] [26]. One of the most recent incarnations of the central database idea can be seen in the IFC Model Server from VTT of Finland [64] designed to host entire building models described in the IAI IFC format.

Within the last three to four years, researchers and commercial application developers in the construction domain have started to develop tools to manipulate complex building models [39]. By storing and managing building information as databases, building information modelling (BIM) solutions can capture, manage, and present data in ways that are appropriate for the user of that data. Because the information is stored in a logically centralised database, any changes in building information data can be logically propagated and managed by software throughout the project life cycle [2] [34]. Building information modelling solutions add the management of relationships between building components beyond the object-level information in object-oriented CAD solutions. This allows information about design intent to be captured in the design process. The building information model contains not only a list of building components and locations but also the relationships that are intended between those objects [37].

This new wave of BIM applications, embody much of the vision of previous academic research such as ATLAS, and COMBINE, whilst still relying on data exchange standards or API level customisation for interoperability/integration. Recently, the American National Institute of Building Sciences has inaugurated a committee to look into creating a standard for lifecycle data modelling under the BIM banner [40]. The 
idea here is to have a standard that identifies data requirements at different lifecycle stages in order to allow a more intelligent exchange of data between BIM enabled applications.

\section{Product data versus knowledge-rich ontology}

This section discusses first the philosophical underpinnings of product data and ontology, and then provides a critical review of their shortcomings, arguing the case for an enhancement of product data to pave the way to more effective, user-friendly conceptualisations of the construction domain through ontology.

\subsection{Philosophical underpinnings of product data and ontology approaches}

Various definitions of what forms an ontology have been formulated and have evolved over time. A good description of these can be found in Corcho et al., (2003) [14]. From the authors' perspective, the best definition that capture's the essence of an ontology is the one given by Gruber, (1994) [28]: "an ontology is a formal, explicit specification of

a shared conceptualization". As elaborated in Studer et al., (1998) [62]:

"Conceptualization refers to an abstract model of some phenomenon in the world which identifies the relevant concepts of that phenomenon. Explicit means that the types of concepts used and the constraints on their use are explicitly defined. Formal refers to the fact that the ontology should be machine processable".

People often find it difficult to see clearly how an "ontology" differs from what they already recognize as a "data model", focussing on the formal nature and structuring mechanisms that seem to be characteristic of both. Certainly, data modelling languages provide the ability to define taxonomies through notations that support classification, 
generalization and specialization, they support the definition of relationships or associations between concepts, and ideas of aggregation and composition, and in terms of these primitives appear to offer the same support for representing concepts and the relationships between them. However, the authors would maintain that trying to understand the distinctions in terms of the modelling primitives that are used is a mistake; it is the nature of the models themselves, the way in which they are derived, and the tools that support their use that provides the differentiation. In order to understand this, it is necessary to return to the underlying problems that make it difficult to achieve a single agreed data model for an industry.

Returning to Gruber's definition of an ontology, a key element is the idea of a shared conceptualization [28]. Typically, in human endeavour, shared conceptualizations are defined over a lengthy period of time, based on the shared experience of a group of people, sometimes referred to as a community of practice [67]. They will involve the definition and use of abstractions that are designed to capture the important aspects of some practical context in order to support a particular activity or type of activity. As such, a shared conceptualization is a socially constructed model or reality that is distinct from reality and is optimized to support the goals and activities of the community of practice in which it was defined. Communities engaged in different activities are likely to form shared conceptualizations that are quite different views of reality, and make up shared "world-views" [12] that provide a basis for highly effective and efficient communications within the respective communities.

In order to understand and formalize the shared world-views of such communities in the form of ontologies to support the integration of diverse human activities, it is important to consider approaches that derive from an interpretive philosophical standpoint rather 
than from a positivist, scientific/engineering one [24]. In such an approach, we try to interpret, accommodate and model what is, rather than trying to change reality to fit a single model. This inevitably results in different ontologies for different communities, but the challenge then is to find ways to allow those communities to collaborate effectively with one another whilst maintaining their existing, efficient, effective separate world-views. The implication is that we are forced to shift our emphasis from developing a standard representation of a single "reality", towards providing mechanisms for supporting communication between differing perceptions of reality, focussing our attention on the overlaps at the boundaries and the specific conceptualizations that are required for such communication to happen.

An important consequence of this shift is that it becomes possible to adopt a more incremental approach to the integration of processes across disciplines. The single data model approach can tend to result in the need for an "all-or-nothing" approach to implementation, and certainly practical issues have been noted regarding the size and manageability of IFC models ${ }^{[4]}$. Even with the existence of product model servers [22], the practical implementation of single-model-based integration seems fraught with difficulty.

\subsection{Alleviating Product Data shortcomings through ontology}

The progress made so far in arriving at the BIM concept and its associated tools is undoubtedly a sizeable step forward in the management, communication and leveraging of construction project information. Both the BIM models used by the commercial vendors and the international standards developed for construction such as STEP, IFC and CIS/2 do however still exhibit shortcomings as highlighted in Rezgui et. al., (1996) [56] and Eastman, (1999) [24] and from our own observations. These shortcomings are 
identified in table 1 along with the level of support offered by both the existing popular standards (including IFCs) and the potential ontology based solutions.

\begin{tabular}{|c|c|c|}
\hline Issue & STEP/IFC & Ontology \\
\hline $\begin{array}{l}\text { Information schema } \\
\text { evolution through time to } \\
\text { support changing project or } \\
\text { industry contexts }\end{array}$ & $\begin{array}{l}\text { Not fully catered for. The IFC } \\
\text { Property Set construct could be } \\
\text { employed to fulfil role for certain } \\
\text { types of information. }\end{array}$ & $\begin{array}{l}\text { This is an inherent attribute of } \\
\text { ontology although not catered for } \\
\text { specifically; but a well-maintained } \\
\text { and updated ontology should } \\
\text { evolve with the domain quite } \\
\text { naturally (whilst respecting and } \\
\text { allowing for business processes } \\
\text { which may require a stable schema } \\
\text { interpretation). }\end{array}$ \\
\hline $\begin{array}{l}\text { Views on data aligned to } \\
\text { user and application needs }\end{array}$ & $\begin{array}{l}\text { Views on STEP models can be } \\
\text { defined in Express-X. The } \\
\text { Application Protocols are } \\
\text { themselves domain specific views } \\
\text { to some extent. Other work is } \\
\text { ongoing to extend models into } \\
\text { specific sub-domains (e.g. } \\
\text { Hassanien Serror et al [31]) }\end{array}$ & $\begin{array}{l}\text { Base level domain ontologies could } \\
\text { be said to be views in their own } \\
\text { right as they support the } \\
\text { information needs of communities } \\
\text { of practice. These may be able to be } \\
\text { transformed through the mechanism } \\
\text { of an upper ontology to suit. }\end{array}$ \\
\hline $\begin{array}{l}\text { Object ownership and rights } \\
\text { management }\end{array}$ & $\begin{array}{l}\text { Not supported, but can be via } \\
\text { EXPRESS }\end{array}$ & Not supported but can be via OWL \\
\hline $\begin{array}{l}\text { Lifecycle management and } \\
\text { placement of data in the } \\
\text { process }\end{array}$ & $\begin{array}{l}\text { STEP defines basic resources } \\
\text { dedicated to relating data to its } \\
\text { place in the process }\end{array}$ & $\begin{array}{l}\text { An ontology of construction would } \\
\text { include concepts describing the } \\
\text { construction process and would } \\
\text { therefore likely feature relations to } \\
\text { classes of data involved in the } \\
\text { process. This would however be a } \\
\text { less fixed notion than the STEP } \\
\text { approach. }\end{array}$ \\
\hline $\begin{array}{l}\text { Recording/embedding of } \\
\text { decision rationale }\end{array}$ & Some basic support [35] & No support \\
\hline $\begin{array}{l}\text { Links to external } \\
\text { information - particularly } \\
\text { unstructured information }\end{array}$ & $\begin{array}{l}\text { Links can be manually defined, but } \\
\text { they have no specific semantics. }\end{array}$ & $\begin{array}{l}\text { Ontologies tend to be built from } \\
\text { text documents using Information } \\
\text { retrieval techniques. The latter are } \\
\text { used to infer external information's } \\
\text { 'relation' to ontology concepts and } \\
\text { by examining other existing } \\
\text { relations, its links to other data. }\end{array}$ \\
\hline
\end{tabular}

Comment [i4]: In response to Reviewer 4 , comment 2

Comment [Y5]: Stefan, can you please
number and add the reference: Kim, J.,
Pratt, M.J., Iyerc, R.G., Srirama, R.D.
Standardized data exchange of CAD
models with design intent, Computer-Aided
Design 2008;40:760-777.

Table 1. Product data versus ontology.

It is clear then that the ontology approach is by no means a cure-all for the ills of product data models as they currently stand. Indeed we would not propose to replace data models so much as enhance them, and the applications used to create and manipulate them, with additional semantic information based on domain and core 
ontologies, as described in the following section. However, we would maintain that ontologies have the right interpretive philosophical underpinning that is more likely to address the information and knowledge sharing requirements of the construction user community as argued in section 4.1

The Web Ontology Language (OWL) is the current leading international standard notation for the definition of ontologies in a machine interpretable fashion. OWL has two primary constructs, namely Classes and Properties. Classes represent categories of things, real or conceptual, and Properties define the relationships between Classes and between instances of those classes. An OWL Class is somewhat analogous to an Express Application Object and whereas Express really only has explicit relationships of the 'is a' inheritance hierarchy type, OWL Properties are far more flexible and explicit in describing a richer set of possible relations between its classes. Many Express relationships are opaquely embedded in the properties of application objects. Similarly, the Express based IFCs define a slightly broader range of relationships but still somewhat fewer than are routinely embedded in an OWL ontology. It is this ability to define rich relationships between Classes that gives an OWL encoded ontology its power.

We see other problems that render data level integration in the STEP or IFC mould less effective than might otherwise be the case. To understand this position it is necessary to consider the way in which data integration mandates a considerable degree of work upfront. This is required in order to agree upon standards, construct a schema for integration, adapt applications to the standards etc. all before any benefits are realised. These issues become more onerous the larger the scope of agreement one is trying to 
achieve (inter-organisational, national, international etc.). Finally, for large international standards efforts, agility is something of a problem. Once the standard is agreed, changing it can take a considerable amount of time, which in an age of rapidly evolving business needs can turn a formerly helpful system into a hindrance [8].

The use of an ontology or multiple ontologies of the construction domain could act as a semantic abstraction layer above current standards and models to further integrate project data in a more intelligent fashion. For example, taking the point on views from table 1 , we believe an ontology with mappings into the underlying data models could be used to provide a more intuitive view of project data for any given actor based on their particular disciplinary concepts and terminology. That same ontology could also provide the view for an actor from a different discipline, based on the relationships explicated within the domain ontologies and between them and the core or upper ontology providing links to the appropriate terminology for the same data items. This type of 'translation' function becomes more compelling when used to view initial project briefs or client constraints and later when viewing the rationale for changes as it helps all actors to understand the reasoning involved in a language they can comprehend easily. Indeed Yang and Zhang, (2006) [71] have proposed extensions to the IFCs to map them into ontologies for the construction domain to improve the semantic interoperability of BIM models in just such a way. The mapping of ontology concepts into the current data model specifications would be performed initially in a semi automated fashion, perhaps using tools such as those identified by Amor, (2004) [1] for mapping between the data standards themselves, suitably modified for the task. 
Taking the schema evolution point from table 1, we would argue for a controlled process for the update and revision of both core and domain ontologies as suggested in Rezgui (2007) [55]. This process of continuous refinement of the ontologies means that at domain level and above, they should evolve naturally with use by the actors in the domains. However, ontologies would not necessarily help with the evolution of the design level information schema (i.e. the drawings or models created by designers), a problem which would remain to be resolved by further research. By contrast, neither STEP nor the IFCs address these issues directly and the relatively static release based versions of the standards limit what might otherwise be a route to domain level evolution to a crawl.

With respect to issues around views over model data, the Express suite of languages employed by the STEP standards include Express-X, which can be used to create so called 'views' of Express based STEP models. These views are essentially new models based on a new schema which has had the elements redundant for the current task or context removed or otherwise transformed by aggregation, decomposition etc. Express$\mathrm{X}$ is used in this scenario to define the way in which the base or input schema[s] (and models based on it) is/are related to the view schema. Language constructs allow for the definition of rules about how to derive objects and properties in the view schema from the input schema[s]. In the authors' opinion, Express-X adds nothing in terms of the internal semantic expressiveness of either the base or view schemas and a dedicated mapping has to be written for each set of base and view schemas.

Where unstructured project information is concerned, the use of ontologies in tandem with other techniques drawn from information retrieval/extraction could be used to 
automatically infer links between the structured and unstructured information and indeed between items of unstructured information, based on the links defined in the ontology. These links lend a greater degree of context to each item relative to the project as a whole. Benefits may also be derived from uncovering previously unseen linkages between various elements of project data using such analysis methods. Kosovac et. al., (2000) [36] and Schere \& Schapke, (2005) [61] have done research work in this or closely related areas. Some elements of the authors' own work have developed or used ontologies in ways similar to these. The eCognos [68] [37] project for example, developed and used a construction oriented ontology to augment the services that it offered as part of the collaborative knowledge management environment also developed on the project. The FUNSIEC project reviewed numerous European semantic resources, compiling them into an educational 'Experience Centre' and further conducting a feasibility study into the production of what the project termed an 'Open Semantic Infrastructure for the European Construction Sector (OSIECS) [3].

\section{Adoption and Diffusion of a Construction Ontology}

In the following section, we discuss the reasons behind the low adoption of IFCs, introduce the requirements for a successful Construction ontology, and provide an illustration of a potential ontology for the sector.

\subsection{Reasons behind the low adoption of IFCs}

A number of studies have been reported in the literature describing various theories and models related to information technology adoption, diffusion, and innovation into the workplace and across industry. Some of these theories describe transition processes and 
mechanisms, including Rogers's stage model of Diffusion of Innovations (DoI) in organizations [60]; whereas others define causality among factors to predict successful transition of a technology, including Davis's Technology Adoption Model (TAM), [16]. TAM argues that end-user acceptance and use of information systems innovations is influenced by their beliefs regarding the technology. In particular, it proposes that perceived usefulness and perceived ease of use influence the use of information systems innovations and that this effect is mediated through behavioural intentions to use [69]. The model highlights the critical role of extrinsic motivation and, in particular, expectations of task-related performance gains in end-users' adoption and use of Information Systems innovations [69]. Roger's Diffusion of Innovation Theory argues that the rate of adoption of a technology is influenced by a number of factors, including: Relative advantage (the degree to which potential adopters see an advantage for adopting the innovation), Compatibility (the degree to which the innovation fits in with potential adopters' current practices and values), Complexity (the degree of the innovation's ease of use), Trialability (the degree to which potential adopters have the availability of "testing" before adopting), and Observability (the degree to which potential adopters are able to see observable results of an innovation).

Considering both models (TAM and DoI) in the context of IFCs, the authors discuss the various factors that may provide an initial explanation to the low adoption of IFCs:

- $\quad$ Perceived usefulness of IFCs: the research community and CAD software industry has failed in convincing the user community about the usefulness in adopting IFCs. This can be attributed to (a) ineffective campaigning and awareness raising from the CAD vendors who tend to be driven by market share and competitiveness concerns; (b) the gap between the Construction IT 
research community and the construction end-users; (c) the nature of the industry which is dominated by a large proportion of SMEs that operate in a survival mode and do not have the resources to consider, investigate, or invest in sophisticated and costly solutions.

- $\quad$ Ease of use of the IFCs (also related to Rogers's complexity factor): the user community has in its majority used CAD to mimic traditional and manual ways of producing drawings. As such, ease of use is intrinsically linked with the complexity associated with the adoption of an object-oriented approach to the production of project documentation. It requires a paradigm shift whereby users have to adopt a lifecycle approach to data and information integration and shift from document (drawing) production to maintaining and enriching a building information model that serves as a basis to generate various consistent documentation, including drawings. The general feeling of the industry is that the adoption of the IFCs would require a steep learning curve.

- $\quad$ Relative advantage: it can be argued that, based on the above facts, the research and CAD vendor community (as they are ultimately the ones who are in charge of implementing the IFCs) have failed in convincing the user community about the advantages resulting from the adoption of the IFCs. In fact, there are limited semantic CAD solutions, and these have been mainly used in research and academic circles. The user community does not therefore see a relative advantage in adopting the IFCs. Indeed, there is a belief that the technology is not yet mature and reliable and that there is an inherent adoption risk.

- Compatibility: this is also a major concern as the IFCs require a paradigm shift and a migration from document centred to building information model 
oriented approaches to design. Real incompatibility concerns have been raised, as there is a lack of preparedness and availability of a full and complete suite of IFC compatible ICT solutions to support the complex design stages.

- Trialability: whilst there are many commercial applications that can import or export IFC format files, their native formats are entirely different and proprietary. Thus trailability is difficult as existing demonstrators are mainly academic prototypes that can hardly be put between the hands of practitioners as (a) these are not stable and robust enough; (b) have mainly been developed with open source or non-industry friendly platforms / solutions (e.g. Object Oriented database systems); (c) require an education / training programme prior to testing so that the users would grasp the underlying concepts.

- Observability: A number of efforts have been made in this respect. For instance, the ATLAS project team in the early nineties have released an interesting video that illustrates the advantages of adopting product data technology. However, this did not reach the whole user community and should have been articulated around a training and education initiative aimed at potential users from large, medium and small construction firms. Instead, this was mainly released and used amongst the research and academic community.

Addressing the above factors should facilitate (a) quicker uptake of a technology, before attention from adopters fades away, and (b) critical mass of adoption, as there must be sufficient users and sufficient software providers that support a standard / technology before it becomes economically viable. Hence, "timing" and "critical mass" emerge as key success factors. 


\subsection{Requirements for a successful Ontology}

The authors would argue that the development of an ontology should factor in the above considerations and, hence, be embedded in a wider initiative aimed at raising awareness of the construction user community through education, training, demonstrations, etc. Also, given the following factors: (a) the fragmented and discipline-oriented nature of the construction sector; (b) the various interpretations that exist of common concepts by different communities of practice (disciplines); (c) the plethora of semantic resources that exist within each discipline (none of which have reached a consensual agreement); (d) the lifecycle dimension of a construction project with information being produced and updated at different stages of the design and build process with a strong information sharing requirement across organizations and lifecycle stages; a suitable ontology development methodology should accommodate the fact that the ontology should be specific enough to be accepted by practitioners within their own discipline, while providing a generic dimension that would promote communication and knowledge sharing amongst these communities.

A critical analysis of the semantic resources available in construction, ranging from taxonomies to thesauri, combined with an understanding of the characteristics of the sector, have helped formulate a set of requirements that ought to be addressed in order to maximize the chances of a wide adoption of any ontology project in the construction sector. These requirements are listed below:

- The ontology should not be developed from scratch but should make as much use as possible of established and recognized semantic resources in the domain. 
- The ontology should be built collaboratively in a multi-user environment: the construction sector involves several disciplines and communities of practice that use their own jargon and have specialized information needs.

- There is a need to ensure total lifecycle support, as the information produced by one actor within one discipline should be able to be used by others working in related disciplines.

- The ontology must be developed incrementally involving the end-users. This is important given the multi-disciplinary and multi-project nature of the industry, and the fact that each project is a one-off prototype.

- The ontology should be flexible and comprehensive enough to accommodate different business scenarios used across projects and disciplines.

- The ontology should be user friendly, i.e., easy to use and providing a conceptualization of the discipline / domain being represented that embeds the technical jargon used in the sector.

- The ontology should be a living system and should allow for future expansion.

\section{3. eCognos: An example of a candidate Ontology for the Construction sector}

Given the above requirements, an ontology was developed, referred to as eCognos [37]; [55]. eCognos is the acronym of an IST Framework 5 project involving the authors that developed a knowledge management platform comprising a number of services centred around an ontology. The latter constitutes one of the key contribution of the project. The eCognos ontology is structured into a set of discrete, core and discipline-oriented, sub-ontologies. Each sub-ontology features a high cohesion between its internal concepts while ensuring a high degree of interoperability between them. These are 
organized into a layered architecture (three layers) with, at a high level of abstraction, the core ontology that holds a common conceptualization of the whole construction domain enabled by a set of inter-related generic core concepts forming the seeds of the ontology. These generic concepts enable interoperability between specialized discipline-oriented modules defined at a lower level of abstraction. This middle layer of the architecture provides discipline-oriented conceptualizations of the construction domain. Concepts from these sub-ontologies are linked with the core concepts by generalization / specialization (commonly known as IS-A) relationships. The third and lowest level of the architecture represents all semantic resources currently available, which constitute potential candidates for inclusion into eCognos either at the core or discipline level.

There are a large variety of available semantic resources that could form the basis for building the eCognos core ontology. These range from classification systems to taxonomies. The latter deserve particular attention as argued in Welty and Guarino, (2001) [66]. One of the principal roles of taxonomies is to facilitate human understanding, impart structure on an ontology, and promote tenable integration. Furthermore, properly structured taxonomies: (a) help bring substantial order to elements of a model; (b) are particularly useful in presenting limited views of a model for human interpretation; and, (c) play a critical role in reuse and integration tasks. Improperly structured taxonomies have the opposite effect, making models confusing and difficult to reuse or reintegrate [66]. IFCs, being more recent and also the closest taxonomy currently in use in the sector, are therefore the preferred candidate semantic resource that can provide the skeleton on which such a core ontology can be built. 
A particular approach is adopted for building and / or expanding the discipline-oriented sub-ontologies. This involves selecting and making use of a large documentary corpus used in the discipline and ideally produced by the end-users. The sub-ontologies are then expanded and built from index terms extracted from commonly used documents using information retrieval techniques [55]. This would allow the effective capture of the jargon and its associated semantics used within each community of practice / discipline. This is illustrated in Figure 3.

Figure 3: Levels of abstraction of the proposed ontology.

An ontology of the construction domain will include concepts devoted to the description of the processes involved in construction projects, thus the relationships between the data on the one hand, and the process within which it is used on the other become more explicit. It is true to say that STEP and the IFCs both define high level schema elements related to process and the use of model data within the process, elements which would need to be duplicated in an ontology. However, certainly where STEP is concerned, those elements are used to describe processes and data use within individual application protocols (APs) and therefore understanding the relationships between data in the individual disciplines (which the APs serve) and the overall project process remains largely unsupported. The explicit links in an ontology between process and data concepts can be used to map out a detailed context for information elements relevant to each actor's role in the project and presented in terms that they would normally use. The existence of a process directly serviced by an information system all of which is ontologically described allows us to create interfaces to the process for individuals based on their role and the specific stage of the process at which they are currently 
working, which present relevant information in a timely manner again customised to the needs and language of the actor.

Therefore, an ontology is not an end in itself. It should have a key and pivotal role in enabling semantic integration across the project lifecycle and its various disciplines. The following sections propose inter-enterprise collaboration architectures and frameworks based on semantic services, underpinned by ontology-based knowledge structures.

\section{Integration through ontology-based semantic services}

The pragmatic ontology approach naturally leads to a service-oriented view of the world, whereby a particular discipline (and therefore the applications that support that discipline) defines a number of services that it may offer to other disciplines, with clearly agreed semantics that can be understood by the communicating parties without having to change the bulk of the conceptualizations that they use in order to provide those services. As such, ontologies should provide conceptualisations that can span several stages of a project while factoring in non-technical (i.e. socio-cultural and organisational) considerations. These conceptualisations can evolve and be adapted to specific contexts that can be shared across services related to one or several design and construction stages. The only changes are related to the applications / services used within each stage.

\subsection{Desktop applications versus web services}


The fundamental difference between a web service and a desktop application is that while the former can be virtually accessed "anytime, anywhere", the latter is only usable from within a desktop or at best through a local / wide area network using a client /server approach. Web services provide a more flexible middleware solution that suits application inter-working, leveraging inter and intra enterprise information systems. Web services are self-contained, web-enabled applications capable not only of performing business activities on their own, but also possessing the ability to engage other web services in order to complete higher-order business transactions [23]. The benefits of web services include the decoupling of service interfaces from implementation and platform considerations, the support for dynamic service binding, and an increase in cross-language and cross-platform interoperability. The challenge of this new form of computing is to move from its initial "Describe, Publish, Interact" capability to support dynamic composition of services into reinvented assemblies, in ways that previously could not be predicted in advance [23][47]. One of the interesting features of web services technology is that it provides the means for traditional desktop applications to be enhanced and upgraded to become and/or to employ web services [47].

Given the shortcomings we have identified in the current product data centric approaches to integration and our suggestion that the use of ontologies at this level could go some way towards addressing them, we would further propose that in order to have systems that actors can interact with in a more intuitive way, ontologies have more roles to play. Here we envisage a number of elementary components, each furnishing a small piece of functionality, usually some discipline specific function, in a fully encapsulated independent fashion. These components, published as Web Services could 
be further composed into higher-level business process components, again selfcontained as per the component based development typical of modern object oriented systems. This model of arbitrary combinations of process components or e-processes as one might call them, allows for greater flexibility in the definition and production of business systems to support construction projects. Ontologies play their role not only at the basic level of a semantic integration layer over the data as detailed previously, but also (a) as a means to describe the concepts and relationships inherent in the processes of construction projects and (b) as a means to articulate at a semantic level the precise nature of an offered service. Working at this higher process oriented level, we begin to see opportunities for resolving some of the lifecycle and context shortcomings of current data models.

The OSMOS [59], C-Sand [69] and eCognos [37] [58] [68] projects in which the authors were involved all employed architectures featuring multiple interoperating services to furnish their functionality to varying degrees. The success of these projects demonstrates the utility of the orchestrated service approach whilst eCognos, as previously mentioned, also featured an ontology to augment its services with semantic capabilities. The systems developed under the eCognos and C-Sand projects also featured the ability to consume arbitrary web services for use 'on the fly'. The development of this feature did not however extend to any automated notion of what those arbitrary services 'were' or 'did', which rather limited its usefulness. Thus we believe that extending the work to encompass services semantically described by means of ontologies would allow for a more automated integration and orchestration to take place, particularly when ontologies are also used to map the services to the business process being served. 


\subsection{Support for semantic e-Processes}

The means by which to aggregate and implement a number of Web Services into a larger business process oriented service are provided through the Business Process Execution Language (BPEL [43]). The BPEL specification defines constructs similar to a simple programming language such as loops, assignments, branches etc, with which to define the flow of calls between a collection of orchestrated services involved in a modelled process.

The whole Web Services stack comprises several other standards including those for securing communications between services and clients (WS-Security), standards for transaction demarcation and management (WS-Transaction) etc. One of the more important amongst the various protocols for the future of Web Services is the Universal Description, Discovery and Integration protocol (UDDI [42]), which allows for the publication and discovery of services on the Web. UDDI has a problem in that whilst it is possible to publish a service description and have it searchable by others, it does not make explicit what the service is for in language that a machine can understand. Thus it is that a human must currently decide whether a particular service is suitable for their business' needs by manually examining both the technical and textual descriptions of a service for compatibility. Removing this manual intervention would allow UDDI to be much more useful than it is today and would permit the type of process oriented services we envisage to be assembled in a more automated fashion. It is here that much current research and development work is concentrated under the Semantic Web Services banner. Standards to describe what a service is for and what the various inputs and outputs actually mean in machine interpretable form are being developed with OWL based ontology for Web Services (OWL-S [44]) and the Web Services Modelling 
Ontology (WSMO [65]). Both of these standards define ontological constructs for describing services and allow external ontologies to be used in the description of service parameters. It is in this role that domain specific ontological concepts such as those defined by the FUNSIEC project [3] or eCognos [37] [55] can be employed to describe services for particular business fields. Together with the lower level Web Services protocols, these ontologies allow for the semi automated composition of aggregate services modelled in line with the business process requirements of specific domains.

While web service technology presents some interesting and promising features, there are a number of issues hindering the wide adoption of this technology, including data quality assurance, quality of service (continuity and recovery plans), as well as a trust, authentication, security, validation, and certification framework (authentication and trust). Addressing these limitations would confer web service technology the industrial robustness that would promote its wide adoption on projects. The next section discusses the dynamic nature of construction project processes and the requirements this places on the proposed e-process enabled computing environment.

\section{Lifecycle dimension and support for the dynamic and long- lasting nature of e-Processes}

The design stage of a project involves interesting examples of long-lasting processes.

This section (a) discusses the limitations of service-based process approaches in addressing the complexity of architectural design, characterised by long-running cooperative processes, (b) provides a comprehensive framework that summarises the above shortcomings, and provides a potential e-Platform solution for the construction industry, and (c) suggests a business model based on the "application service provider 
(ASP) model" for the deployment of the e-Platform solution for the construction industry.

\subsection{Design process characteristics}

The multiplicity of circumstances governing the decision making processes inherent in architectural design leave scope for numerous misunderstandings, unforeseen difficulties created by inappropriate or ill-conceived information, changes and decisions which fail to propagate amongst all interested parties. Further, these circumstances are commonly compressed into short timeframes featuring periods of intense activity in which many decisions are made. Moreover, design is a predictive activity, that has to be planned and instrumented, and for which actions that will be implemented are defined beforehand. At the same time, design is a reactive activity that evolves and adapts as its content changes with the environment and with the personality of the actors that conduct it. All the complexity of the design therefore lies in this duality. It is therefore agreed upon that if design steering consists of organizing and planning tasks with already identified mechanisms and results; it also consists in managing events, actions and situations that are not initially known and formalized. The success or failure of a project is often explained by the manner in which these different unplanned situations are managed and controlled.

The design process is currently supported by a number of software applications, including CAD and related engineering software. It can be modelled as a dynamic, long-lasting, process. However, there exist several limitations of service process approaches that hinder the effective adoption of such a paradigm. Long running cooperative processes are subject to evolutions and changes of differing nature: process 
model evolution due to change in the environment (change in the law, change in the methodology), process instance evolution (or ad-hoc evolution) due to specific events occurring during a given process execution (delay, newly available or missing resources) or partnership evolution at execution time having an impact on part of the process. These shortcomings require essential advances and improvements, including:

- Tracking of history of changes: change management is an important issue in long lasting processes. When a process model (or an abstract process) is changed, it may be important to migrate running processes to reflect these changes. However, this migration is sometimes only feasible under certain conditions, and must be implemented dynamically.

- Partner change during process execution (dynamic change of partner, with partial fulfilment of choreography): during a long lasting process, a partner may fail to complete a conversation, or even disappear. In this case, a new partner has to be selected, dynamic re-composition has to occur and part of the execution may have to be restarted. It is essential that change of partners be dynamically supported in the context of the executed choreography.

- Partial rollback (check pointing): events such as dynamic change of partners may require a process to be partially rolled back and re-executed with a new partner. This partner may even benefit from the previous partial execution. Partial rollback or compensation may also be triggered by a change in the process. These scenarios are unsupported or ill-supported by the current BPEL specification. Partial rollback will require adapting the Business Activity transaction model to allow a more flexible approach than the simple open nested transactions. 
- Process evolution (unpredictable event management, dynamic process evolution): during a long lasting process, events may occur such as unexpected delay or resources evolutions that require more or less important changes in the process. These changes have to be done while ensuring the correctness of the process itself. The kind of changes that have to be tackled concern adding or removing operations in the process, change in the ordering of the steps, changes in the relationships with the partners (policy evolution). Some work regarding dynamic process evolution has been done already in the area of workflow management systems, which would be of benefit if adapted to BPEL processes. Ad hoc changes are required to ensure the reliability and the validity of the resulting process.

BPEL as it is defined does not support these kinds of process model evolution and even less ad-hoc evolutions. This is a real problem for long running processes as experienced during the design stage of a project where external and internal unexpected events may require adaptation and evolution. It is worth noting the pivotal role of a construction ontology in resolving many of the above limitations, in particular those related to semantic compatibility between services (as illustrated in section 7). Since individual web services are created in isolation, their vocabularies are often rife with problems having abbreviations, different formats, or typographical errors. Furthermore, two terms with different spellings may have the same semantic meaning, and thus are interchangeable. Diverse matching schemes have been developed to address these semantic resolution problems, including in the context of the eContent FUNSIEC project [3]. In general, matching approaches may fall into three categories: (a) Exact match using syntactic equivalence; (b) Approximate match using distance functions (TF-IDF, 
Jaccard, SoftTF-IDF, Jaro, or Levenstein distance); and (c) Semantic match using ontologies. The latter is the authors' preferred approach as it provides the possibility to reason about web services, and to automate web services tasks, like discovery and composition [50].

\subsection{Proposed e-Platform solution}

Figure 4 illustrates a comprehensive framework that summarises the above shortcomings and issues identified in the paper, and provides a potential e-Platform solution for the construction industry. This has been inspired from the authors' research. The underlying web-service infrastructure has already been developed as reported in [52], while some of the suggested services have already been specified and prototyped, including the Ontology service [69] and the Semantic compatibility service [50]. A brief explanation of each service is given below:

- Semantic compatibility service: this, as described earlier, determines whether two services can inter-work prior to invocation. In essence, the semantics of their underlying data structures are looked up in an ontology and semantic relatedness checks are performed.

- Process monitoring service: this provides a means to monitor the various states a process goes through and anticipate potential problems, including availability of services.

- Ontology service: this provides the functionality required to make the selected ontology available to the other services, which may require it. It provides an interface to query the ontology, including its concepts, and semantic relationships. 
- Intelligent resource discovery service: This service makes use of a categorisation sub-service that provides a context and criteria-based categorisation of the information held within the UDDI registry for effective use and mining by potential business partners. It provides a standardised interface to query and make use of the Intelligent Categorised UDDI Registry generated through the categorisation sub-service.

- Composition service: this provides the interface that enables the modelling of an e-Process. This is achieved through the composition of discrete services.

- Process execution service: this is used to execute a modelled e-Process through the composition service, and provides an interface to control and interfere with its execution following problems identified by the process monitoring service.

\subsection{A proposed business model}

We would also suggest a business model based on the application service provider model for the deployment of the technical solution. Here we envisage three roles as described in [8]:

- Service provider - any organisation having services (specifically web services) that they wish to monetise and offer to third parties for their consumption. Providers register their services with the aggregator for publishing and composition into e-Processes.

- Service aggregator/host - An organisation with responsibility for hosting the infrastructure defined in the middle layer of figure 5 below. The aggregator composes business systems (e-Processes) from the offerings of registered 
service providers tailored to the requirements of particular projects or organisations (real or virtual) and their business processes.

- Service client - any organisation requiring business system functionality to support their business processes.

It is believed that this model will aid construction sector SMEs in adopting technology which may otherwise be out of their reach either technically or financially. We do not however prescribe who may take on the individual roles and indeed envisage that single organisations, particularly large technologically sophisticated ones, might encompass elements of all of them. Centralising infrastructure in this way has the additional benefit of providing a single point of contact for service, support and legal/contractual issues from the point of view of service clients.

Figure 4: An Ontology-based Framework for e-Process Execution and Management.

\section{Preliminary roadmap for semantic service-based e- Construction}

The construction industry is different from other large industries since it relies on a very high proportion of SMEs involved in both off-site management, design and procurement and on-site fabrication services. Such SME companies tend only to be present in a project during their part of the activity and this discontinuity of involvement is a particular challenge for the industry in relation to the adoption of the right business models and modes of project operation. Certainly the industrialisation of construction cannot be done in exactly the same way as in other sectors. This section proposes an initial roadmap for the adoption of the proposed approach. Key features of the industry 
are provided, followed by a proposal for an alliance model. An initial staged roadmap is then formulated.

\subsection{A proposed alliance model for the industry}

The general picture of construction is therefore of an industry that is a pyramid with control being in the hands of large players with a large base of SMEs relatively weak in influencing the early important decisions in projects because they are usually not appointed and in place. The expertise of SMEs cannot therefore be brought to bear at the conceptual and feasibility stages in construction. Also, some industry major players are reducing the circle of specialists and sub-contractors they use. This has an impact on the supply/value chain by forcing the emergence of transient knowledge based alliances. However, these are highly likely to be dominated by large industry players, where SMEs have no influential role. Hence, SMEs should anticipate this trend by forming cooperative transient production networks [30].

The way the authors view the future is for SME alliances to be established, forming knowledge infused virtual networked organisations that can "punch at a higher weight" than the individual SMEs. The alliance brings together SMEs in a relationship of trust to provide holistic competence to a field of activity - for instance, "energy consumption, energy performance monitoring and integration of energy resources". The alliance would market itself with this capability and ultimately achieve a recognised industry "branding" much as a "big name" player. Moreover, because the alliance SMEs would work frequently together (though not exclusively), they can (a) standardise processes and information flows, (b) improve sharing of information and re-use of best practice, (c) create value out of the knowledge possessed across the alliance, thus making 
investment in collaborative technologies highly attractive and productive. A key function in an alliance will be to broker, manage and integrate the alliance acting as the conduit with clients and managing the quality of solutions achieved. The authors view an SME alliance as a kind of Virtual Factory, with different alliance members contributing their often much specialised knowledge on particular aspects to virtually configure a "best" solution against requirements and regulations.

Thus, the authors argue a transformation of production business models for

Construction SMEs, to achieve economies of scale in the production of standardised processes and product approaches with economies of scope in various stages of assembly in order to provide flexibility to satisfy customer choices [49].

This is where service-oriented architectures have the ability to provide the underpinning middleware infrastructure that can support the operations of virtual alliances.

Ontologies will have a pivotal role in this, as they will provide the semantic backbone to enable concept integration between actors and applications.

\subsection{An initial adoption roadmap}

E-processes are typically designed, developed, and deployed by enterprises that want to compose internal capabilities with third-party capabilities, either for internal use or to expose them as (complex, value-added) e-services to customers. For both e-processes and e-services, the need for companies to expose internal details of how they run their business is not envisioned. Still, companies should be able to express, in a standard format, the interaction aspects for their service offer as well as for their service needs. A common language is crucial for the assessment of the compatibility between the

Comment [Y6]: Stefan, can you please number and add the reference: Rezgui, Y. and Miles, J.C. Transforming SME strategies via innovative transient knowledge-based alliances in the Construction Sector, In: Proc. 2009 7th IEEE International Conference on Industria Informatics. Cardiff, 24 - 26 June 2009.

common language is crucial for the assessment of the compatibility between the 
interaction processes offered by an e-service provider and those expected by the designer of an e-process. The effectiveness and efficiency of business processes impact directly the profitability of a company. It is in the best interest of e-service providers as well as e-service consumers to understand the operational requirements for their cooperation. This can best be achieved in the context of an alliance. However, as traditional processes are designed around the operational model of customised business applications, e-processes should be designed around e-services. A clear understanding of the business interaction model of an e-service is paramount, and this should be facilitated by the service provider (see section 8.3). Migrating to e-Processes involves the three following stages illustrated in Figure 5 and briefly described below: 
- Phase 1: integration of existing internal assets. Enterprises (including SMEs) that form an alliance should align their processes to the business needs and vision of the alliance and work towards reducing costs and improving business process execution quality and speed. Process automation and management technologies enable the separation of business, resource and application logics. Processes can be controlled, managed, and evolved separately from the applications. In this phase, resources remain internal to the enterprise.

- Phase 2: static integration with partner processes on a case-by-case basis. By incorporating e-services provided by business partners into an e-process (within or across alliances), an enterprise can create processes that utilize external resources offered by other alliance members. However, in this phase, service selection and invocation is still performed in an ad-hoc way, and requires preliminary agreements (from business, legal, and technical perspectives) between the cooperating alliance companies.

- Phase 3: dynamic integration with negotiation, with companies. Beyond such static use of external services, fully dynamic e-processes make decisions each time they are executed in order to invoke the best available service that can fulfil the customer's needs. The traditional design-deploy cycle of phases 1 and 2 is changed to e-Processes that cannot be anticipated in advanced. This will make full use of the approach proposed in the paper, centred around the use of ontology.

Figure 5: A proposed preliminary roadmap. 
However, for the above roadmap to work, the construction IT and software (including CAD) vendor communities should play a key role in delivering a robust base technology (service infrastructure), supported by key adopters (including engineering design consultants and contractors), and work in unison towards addressing the identified factors that have affected the wide adoption of previous initiatives, including: relative advantage (the degree to which potential adopters see an advantage for adopting the innovation), compatibility (the degree to which the innovation fits in with potential adopters' current practices and values), complexity (the degree of the innovation's ease of use), Trialability (the degree to which potential adopters have the availability of “testing" before adopting), and Observability (the degree to which potential adopters are able to see observable results of an innovation). It is worth re-emphasising the importance of "timing" and "critical mass" in ensuring the successful deployment and adoption of this technology, as argued earlier in the paper.

\section{Conclusion}

It has been argued that, for successful integration in construction through IT, attention needs to be paid to supporting processes through service oriented approaches; and to improving the human communication aspects and migrating existing information systems through work on ontologies. Rather than attempting to create a vision of common data standards that need to be achieved in whole for benefits to be seen, these approaches provide the potential to realize incremental benefits for the industry through progressive automation of processes, which may be more palatable to the industry. In this scenario, existing work on product models is no longer directed towards the exchange of complete data sets between applications, but provides the cornerstone for defining services that fit into a service-oriented architecture to support construction processes; for defining ontologies that can help to integrate and migrate valuable, 
existing, unstructured information and knowledge; and for focussing directly on the interactions between different human actors and disciplines in the construction industry.

A technological solution, it has been shown, has to demonstrate capability of supporting the central project (including design) business processes, allow integration of systems and interoperability between disparate applications and enable the management of interactions between individuals and teams, whilst at the same time taking into account the fact that the industry is dominated by SMEs and operates within tight financial margins. The proposed approach will essentially provide a scalable and user friendly environment to support teamwork in the sector by: (a) delivering to clients customised solutions in the form of web services maintained by a dedicated application service provider [8]; (b) providing an alternative to the traditional licensing model for software provision by introducing a model based on service rental or offered on a pay-per-use basis [52] [8]; (c) providing a change of focus from " point to point"' application integration to service collaboration and inter-working; (d) delivering higher-order functionality, composed from elementary services, providing direct support for business processes; (e) providing a ubiquitous dimension to business processes, as services can be invoked anytime, anywhere from a simple web-browser; (f) enabling a single point of contact for service and client support.

The paper argues that ontologies provide a richer conceptualisation of a complex domain such as construction compared to existing product data standards. An ontology should be viewed as a living system. The issue of the existence of a unique ontology for an entire sector remains open. This suggests that while the eCognos Core Ontology forms a robust basis for interoperability across the discipline-oriented ontologies, the 
latter will need adaptation and refining when deployed into an organization and used on projects. Another issue that can be raised is that related to the adoption of user specific views or perspectives on the global ontology. In fact, in many instances, some actors might be required as part of their job to deal with more than one discipline ontology to conduct a task. This necessitates some flexible mechanisms that can enable the rapid combination of two or more discipline ontologies into a single view / perspective. It is hoped that the paper will stimulate thinking and discussion about the evolution of data products to knowledge-rich ontologies, and their use in the context of construction projects to support seamless eProcesses.

It is envisaged that the technology solutions (i.e. ontology and service architecture) as well as the adoption and diffusion issues discussed in the paper can be furthered and extended to other industry applications and sectors. These form the research efforts in which the authors are currently engaged, and will be reported in related publications.

\section{Acknowledgements}

The authors would like to acknowledge the financial support from the European Commission under the IST and eContent programs, as well as the EPSRC on the ongoing EP/E001882/1 grant. 


\section{References}

[1] Amor, R.W. Supporting standard data model mappings. Proceedings of EC-PPM 2004: 35-40, Istanbul, Turkey, 8-10 September, 2004.

[2] Autodesk. Building Information Modelling, 2007 available on-line at http://www.autodesk.com/buildinginformation/

[3] Barresi, S., Rezgui, Y., Lima, C. and Meziane, F. Architecture to support semantic resources interoperability in Interoperability Of Heterogeneous Information Systems Proceedings of the first international workshop on Interoperability of heterogeneous information systems, Bremen Germany, November 4th $2005: 79-82$

[4] Bazjanac, V. Building energy performance simulation as part of interoperable software environments. Building and Environment 2004, 39(8): 879-883.

[5] Björk, B-C., RATAS Project - Developing an Infrastructure for ComputerIntegrated Construction. Journal of Computing in Civil Engineering 1994; 8, (4) : 400419.

[6] Björk, B.-C. and Penttilä, H. A scenario for the development and implementation of a building product model standard. Advances in Engineering Software 1998; 11 (4): 176-187.

[7] Björk, B.-C. Basic structure of a proposed building product model. Computer-Aided Design 1998; 21 (2): 71-78.

[8] Boddy, S., Rezgui, Y., Cooper, G.S. and Wetherill, M. Computer Integrated Construction: A Review and Proposals for Future Direction. Advances In Engineering Software 2007; 38(10): 677-687.

[9] Bohms, M., Tolman, F. and Storer, G. ATLAS, A STEP Towards Computer Integrated Large Scale Engineering. Revue Internationale de CFAO 1994; 9(3): 325337. available on-line at: http://www-uk.research.ec.org/esp-syn/text/7280.html

[10] Bosch, K. O., Bingley, P., and van der Wolf, P., Design flow management in the NELSIS CAD framework. In Proceedings of the 28th Conference on ACM/IEEE Design Automation 1991 (San Francisco, California, United States, June 17 - 22, 1991).

[11] Brown, A. Rezgui, Y. Cooper, G. Yip, J. and Brandon, P. Promoting Computer Integrated Construction Through the Use of Distribution Technology. ITcon 1996; 1: 51-67.

[12] Checkland, P. and Scholes, J. Soft Systems Methodology in Action 2000. John Wiley \& Sons, Inc, New York.

[13] Cooper, G. Cerulli, C. Lawson, B. R. Peng, C. and Rezgui, Y.Tracking decisionmaking during architectural design, ITcon 2005; 10: 125-139, http://www.itcon.org/2005/10. 
[14] Corcho, O. Fernando-Lopez, M. Gomez-Perez, A. Methodologies, tools and languages for building ontologies. Where is their meeting point? Data and Knowledge Engineering 2003; 46: 41-64.

[15] Daniell, J. and Director, S. W. An object oriented approach to CAD tool control within a design framework. In Proceedings of the 26th ACM/IEEE Conference on Design Automation (Las Vegas, Nevada, United States, June 25 - 28, 1989).

[16] Davis, F.D., 1993. User acceptance of information technology: system characteristics, user perceptions and behavioural impacts. International Journal of ManMachine Studies 1993, 18 (3): 475-487.

[17] Dubois, A.M., Flynn, J., Verhoef, M.H.G. \& Augenbroe, F. Conceptual Modelling Approaches in the COMBINE Project. presented in the COMBINE final meeting 1995, Dublin. http://erg.ucd.ie/combine/papers.html

[18] Eastman C.M. Modelling of buildings: evolution and concepts. Automation in Construction 1992; 1: 99-109.

[19] Eastman, C. Wang, F. You, S.-J. and Yang, D. Deployment of an AEC industry sector product model. Computer-Aided Design 2004; 37 (12): 1214-1228.

[20] Eastman, C.M. Information Exchange Architectures for Building Models, in Lacasse, M.A. \& Vanier, D.J. (eds.) Durability of Building Materials and Components 8, Institute for Research in Construction, Ottawa, Canada; p. 2139-2156.

[21] Ellis, J.H.M. and Kiely, J.A. Action Inquiry Strategies: taking stock and moving forward, Journal of Applied Management Studies 2000; 9(1): 83 - 94.

[22] Estman, C.M. Building Product Models, CRC Press, 1999, Boca Raton, FL, USA.

[23] Ferris, C., Farrell, J. What are web services. Communications of the ACM 2003; 46 (6): 31.

[24] Fitzgerald, B. and Howcroft, D. Competing dichotomies in IS research and possible strategies for resolution. In Proceedings of the international Conference on information Systems (ECIS), 1998, Helsinki. pp. 155-164.

[25] Froese, T. and Paulson, B. (). OPIS, an object-model-based project information system. Microcomputers in Civil Engineering 1994; 9:13-28.

[26] Giannini, F. Monti, M. Biondi, D. Bonfatti, F. and Daniela Monari, P. A modelling tool for the management of product data in a co-design environment. Computer-Aided Design 2002, 34 (14): 1063-1073.

[27] Gielingh, W.F.General AEC Reference Model.Tech. Rep. 1988, P.O. Box 462600 AA, the Netherlands,BI-88-150, October. 
[28] Gruber, T. Towards principles for the design of ontologies used for knowledge sharing. International Journal of Human Computer Studies 1994; 43(5/6): 907-928.

[29] Gu, P. and Chan, K. Product modelling using STEP. Computer-Aided Design 1995; 27 (3): 163-179.

[30] Hagel, J. and Armstrong, A. G. 1997. Net gain - expanding markets through virtual communities. Harvard Business School Press, Boston, Massachusetts.

[31] Hassanien Serror, M., Inoue, J., Adachi, Y. and Fujino, Y. Shared computer-aided structural design model for construction industry (infrastructure). Computer-Aided Design, 2008; 40(7): 778-788.

[32] IAI. International Alliance for Interoperability. IAI Web Site, web page at http://www.iai-international.org [accessed July 15, 2007].

[33] ISO 10303-1:1994 Industrial automation systems and integration -- Product data representation and exchange -- Part 1: Overview and fundamental principles. International Standards Organization 1994. TC 184/SC 4 available on-line at: http://www.iso.ch/cate/d20579.html

[34] Kam C, Fischer M, Hänninen R, Karjalainen A and Laitinen J. The product model and Fourth Dimension project, Special Issue IFC - Product models for the AEC arena. ITcon 2003; 8: 137-166.

[35] Kim, J., Pratt, M.J, Iyer, R.G. and Sriram, R.D. Standardized data exchange of CAD models with design intent. Computer-Aided Design, 2008; 40(7): 760-777.

[36] Kosovac, B., T.M. Froese, and D.J. Vanier (). "Integrating Heterogeneous Data Representations In Model-Based AEC/FM Systems", Construction Information Technology 2000, Proceedings of CIT 2000 - The CIB-W78, IABSE, EG-SEA-AI International Conference on Construction Information Technology, Reykjavik, Iceland, 28-30 June, 2000, G. Gudnason (Ed.), Icelandic Building Research Institute 2000; 2: 556-567.

[37] Lima, C., El-Diraby, T. and Stephens, J. Ontology-Based Optimisation of Knowledge Management in e-Construction. ITcon 2005; 10: 305-327.

[38] M. Fischer, T. Hartmann, E. Rank, F. Neuberg, M. Schreyer, K. Liston, J. Kunz Combining different project modelling approaches for effective support of multidisciplinary engineering tasks, in: P. Brandon, H. Li, N. Shaffii, Q. Shen (Eds.), INCITE 2004 - International Conference on Information Technology in Design and Construction, Langkawi, Malaysia, 2004:167-182.

[39] Mannisto, T. Peltonen, H. Martio, A and Sulonen, R. Modelling generic product structures in STEP. Computer-Aided Design 1998; 30(14): 1111-1118.

[40] Meziane, F. and Rezgui, Y. Document management methods based on similarity contents. Information Sciences 2004; 158: 15-36. 
[41] NIBS. National Institute of Building Sciences. BIM Committee Web site at http://www.nibs.org/BIMcommittee.html [accessed 13/07/2007].

[42] OASIS. OASIS UDDI V3.0 Specification. web page at http://uddi.org/pubs/uddi_v3.htm [accessed 17/07/2007]

[43] OASIS. Web Service Business Process Execution Language Technical Introduction. available at http://www.oasisopen.org/committees/download.php/23068/WSBPEL\%20Technical\%200verview\%20for\%20Developers\%20and\%20Architects\%20\%20Part\%201\%20\%28Frank\%20Ryan\%29.pdf [Accessed 17/07/2007]

[44] OWL-S Coalition. OWL-S Coalition web site. web page at http://www.daml.org/services/owl-s/1.0/ [accessed 16/07/2007].

[45] Rezgui, Y. (b) Role-Based Service-Oriented Implementation of a Virtual Enterprise: A Case Study in the Construction Sector. Computers in Industry 2007; 58(1): 74-86.

[46] Rezgui, Y. (c) Knowledge Systems and Value Creation: An Action Research Investigation. Industrial Management and Data Systems 2007; 107(2):166-182.

[47] Rezgui, Y. and Medjdoub, B. A Service Infrastructure to Support Ubiquitous Engineering Practices. the 8th IFIP Working Conference on Virtual Enterprises 2007, Guimaraes, Portugal.

[48] Rezgui, Y. and Meziane, F. A Web Services Implementation of a User Centred Knowledge Management Platform. Journal of Intelligent Information Technologies $2005 ; 1(4)$

[49] Rezgui. Y. and Miles, J.C. Transforming SME strategies via innovative transient knowledge-based alliances in the Construction Sector, In: Proc. 2009 7th IEEE International Conference on Industrial Informatics. Cardiff, 24 - 26 June 2009.

[50] Rezgui, Y. and Nefti-Meziani, S. Ontology-Based Dynamic Composition of Services Using Semantic Relatedness and Categorisation Techniques. ICEIS: 9th International Conference on Enterprise Information Systems, 12-16, June 2007, Funchal, Madeira - Portugal.

[51] Rezgui, Y. Cooper, G. A Proposed Open Infrastructure for Construction Project Document Sharing. ITcon 1998; 3:11-25.

[52] Rezgui, Y. Exploring Virtual Team-Working Effectiveness in the Construction Sector. Interacting with Computers 2007; 19(1): 96-112.

[53] Rezgui, Y. Ontology Driven Knowledge Management Using Information Retrieval Techniques. Computing in Civil Engineering 2006; 20(3).

[54] Rezgui, Y. Review of Information and Knowledge Management Practices State of the Art in the Construction Industry, Knowledge Engineering Review 2001; 16(2). 
[55] Rezgui, Y. Text Based Domain Ontology Building Using tf-idf and Metric Clusters techniques. Knowledge Engineering Review, 2007, 22(4), 379-403.

[56] Rezgui, Y., Brown, A., Cooper, G., Aouad, G., Kirkham, J. and Brandon, P. An Integrated Framework for Evolving Construction Models. The International Journal of Construction Information Technology 1996; 4(1): 47-60.

[57] Rezgui, Y., Cooper, G. and Brandon, P. Information management in a collaborative multiactor environment: the COMMIT approach. Computing in Civil Engineering 1998; 12 (3):136-44.

[58] Rezgui, Y., Zarli, A. Paving the way to digital construction: a strategic roadmap. Journal of Construction Management and Engineering (Journal of the American Society of Civil Engineering) 2006; 132(7): 767-776.

[59] Rezgui, Y., Zarli, A., Bourdeau, M. and Cooper. G. Inter-Enterprise Information Management in Dynamic Virtual Environments: The OSMOS Approach. In Proceedings of the CIT2000 - The CIB-W78, IABSE, EG-SEA-AI International Conference on Construction Information Technology, 2000, 28-30 June, Reykjavik: 731-741.

[60] Rogers, E.M. Diffusion of Innovations, fourth ed. 1995. Free Press, New York.

[61] Scherer, R. J., Schapke, S.-E. Constructing Building Information Networks from Proprietary Documents and Product Model Data. In proceedings of cib-w78 2005 22nd Conference on Information Technology in Construction, Scherer R.J, P. Katranuschkov \& S.-E. Schapke (ed.): 343-348.

[62] Studer, R., Benjamins, V., and Fensel, D. Knowledge engineering: Principles and methods. IEEE Transactions on Data and Knowledge Engineering 1998; 25: 161-197.

[63] Turner J. AEC Building Systems Model, working paper ISO/TC/184/SC4/WG1, October 1988.

[64] VTT. IFC Model Server. http://ve.cic.vtt.fi/IMSvr/index.html

[65] W3C. Web Services Modelling Ontology. W3C Member Submission, W3C Web site at http://www.w3.org/Submission/WSMO/ [Accessed 16/07/2007]

[66] Welty, C. and Guarino, N. Supporting ontological analysis of taxonomic relationships. Data and Knowledge Engineering 2001; 39(1): 51-74.

[67] Wenger, E. Snyder, W. Communities of practice: the organizational frontier. Harvard Business Review 2000, Jan-Feb: 139-145.

[68] Wetherill, M. Rezgui, Y., Lima, C. and Zarli, A. Knowledge management for the construction industry: the eCognos project. Special Issue ICT for Knowledge Management in Construction, ITcon 2002; (7): 183-196. 
[69] Wetherill, M., Rezgui, Y., Boddy, S. and Cooper, G.S. Intra- and Interorganizational Knowledge Services to Promote Informed Sustainability Practices. Journal of Computing. in Civil Engineering 2007; 21,(2): 78-89.

[70] wsmo.org WSMO Registry working draft, April 2004 available at http://www.wsmo.org/2004/d10/v0.1/ [Accessed 05/07/2008]

[71] Yang, Q.Z. and Zhang, Y. Semantic interoperability in building design: Methods and tools. Computer-Aided Design 2006; 38: 1099-1112. 